\title{
Considering Drug Metabolism and Molecular Complexity in Drug Design
}

\author{
Rüdiger Hardeland* \\ Department of Zoology and Anthropology, University of Göttingen, Germany
}

Submission: August 16, 2017; Published: October 04, 2017

*Corresponding author: Rüdiger Hardeland, Johann Friedrich Blumenbach Institute of Zoology and Anthropology, University of Göttingen, Bürgerstr, 50, D-37073, Göttingen, Germany, Tel: +49-551-395414; Email: rhardel@gwdg.de

\begin{abstract}
Drugs design is usually focused on affinity to binding sites. However, such an approach, which is meaningful and promising, should not neglect the contribution of metabolism to the efficacy and tolerability of a drug. Metabolism is often primarily regarded as a source of drug elimination. On the other hand, metabolites can have their own pharmacological properties, which may be highly undesired, but, in other cases, can be favorable. In this short review, examples are given for oxidotoxicity of metabolites, including the possibility of organic redox cycling, for non-enzymatic formation of metabolites with undesired properties, for unexpected changes in lipophilicity, and for presumably favorable properties of certain metabolites. The relationship of molecular complexity of a drug to the number of metabolites is addressed. The necessity for not neglecting the possible toxicity of minor metabolites is emphasized. Drug metabolism should be more systematically included in drug design, exceed actions of cytochrome P450 isoforms and also focus on metabolite properties.
\end{abstract}

Keywords: Agomelatine; Berberine; Indoles; Ramelteon; Redox cycling; Tasimelteon

\section{Introduction}

In the last years, considerable progress has been made concerning efficient drug design by in silico adapting drug molecule candidates to known binding sites that had been spatially elucidated by methods of structural biology. This allows identification of suitable ligands with regard to both geometry of the binding pocket and affinity as deduced from knowledge of docking points and energy calculations. Other, more conventional approaches used especially in cases in which the structure of the binding site has not been sufficiently characterized are based on modification of existing ligands. Both procedures yield molecules that bind with reasonable affinity to the target proteins. However, after having identified a suitable compound, other important information is required and not always easily obtained, in spite of high relevance to the tolerability of a drug. One problem may consist in the undesired binding to non-target proteins, which may result in side effects. A further aspect of relevance that may be associated with difficulties of future application can arise from drug metabolism. Some aspects may already be deduced from experience, but in many cases, the parent compound is too complicated to foresee all possibilities of conversion into other compounds. In part, metabolic routes may be esteemed on the basis of knowledge on properties of cytochrome P450 (CYP) enzymes. However, this is already complicated by the high number of different CYP isoforms. Moreover, additional enzymes have to be considered, too, especially those catalyzing other oxidation or dioxygenation reactions. Finally, non-enzymatic reactions may sometimes need particular attention, since free radicals may cause dimerization or even oligomerization of sufficiently reactive compounds, as studied in detail in several indoles $[1,2]$ and, thereby, generate unfavorable metabolites. As a general rule, the number of possible metabolites is increasing with the size and the complexity of a drug molecule. In particular, this is the case if a drug is composed of several ring systems, especially when connected by aliphatic bridges. This is most evident in multifunctional hybrid molecules.

In this short review article, possible problems arising from metabolism shall be addressed using examples from the field of natural and synthetic aromates with multiple ring systems. The message of these considerations concerns the insufficiency of conducting toxicity tests only with the drug itself and the importance of identifying the metabolites and screening them for toxicity as well as for their persistence in the body.

\section{Oxidotoxicity by drug metabolites}

It is of utmost importance to be aware of the possible oxidotoxicity of drug metabolites, especially when deriving from aromates. Many drugs are metabolized by CYP enzymes. Apart from the possibility of dealkylation, they mainly catalyze hydroxylation reactions, sometimes also epoxide formation. If the substrate is already a hydroxylated aromate, the dihydroxylated product may undergo non-enzymatic reactions that generate hydrogen peroxide (H2O2), thereby forming a 
quinone. This is well-known since long from dopamine [3], but is applicable to other dihydroxylated aromates as well and may be overlooked if the possibility of generating a dihydroxylated drug metabolite has not been considered. In the case of the dopamine o-quinone, local enrichment of $\mathrm{H} 2 \mathrm{O} 2$ leads to the formation of the highly toxic 6-hydroxydopamine quinone, which can be alternately formed via hydroxylation to 6-hydroxydopamine and subsequent quinone formation, thereby again generating $\mathrm{H} 2 \mathrm{O} 2$ [3]. The amounts of $\mathrm{H} 2 \mathrm{O} 2$ formed under these conditions may be less relevant to damage of other biomolecules, but they are certainly of importance for enhancing the local production of toxic secondary metabolites. Insofar the argument that the amounts of $\mathrm{H} 2 \mathrm{O} 2$ are small relative to those generated in other, e.g., mitochondrial processes is not convincing.

An additional possibility that has to be taken into consideration is that of organic redox cycling. For instance, this has been assumed to occur in the case of the synthetic melatonergic drug agomelatine [4]. This naphthalenic compound, chemically designated as $\mathrm{N}$-[2-[7-methoxynaphth1-yl)ethyl] acetamide (CAS 138112-76-2), does not contain a hydroxyl group, but its methoxy residue can be dealkylated by CYP2C9. Further hydroxylation leads to a dihydroxylated compound, which can be alternately formed via hydroxylation of agomelatine by, e.g., CYP1A1, followed by the aforementioned dealkylation. Another o-dihydroxylated metabolite can be generated by epoxide formation catalyzed by CYP1A2, followed by non-enzymatic cleavage of the epoxide. By interacting with other redox-active interaction partners, the dihydroxylated metabolites, which may also be referred to as naphthohydroquinones, can undergo organic redox cycling known for this class of compounds, thereby generating superoxide anions $(02 \bullet-)$, which are further converted to other reactive oxygen species [4]. The cycling between the respective naphthohydroquinone and the naphthosemiquinone as well as between the naphthosemiquinone and the naphthoquinone can be driven by ascorbate, which, in this case, like in other forms of redox cycling behaves, in the balance, as a strong prooxidant, contrary to its antioxidant action under noncycling conditions [4]. Other antioxidants may, instead of ascorbate, likewise participate in driving the redox cycle. One of the consequences of redox cycling by naphthoquinones is the depletion of reduced glutathione. With reference to agomelatine and its hydroxylated metabolites, the necessity of considering a redox-based toxicity of naphthalenic compounds had been addressed relatively soon after its approval [5]. In some patients treated with agomelatine, the drug proved to be hepatotoxic, sometimes severely [4, 6,7], whereas the original publications had stated a good tolerability. Hepatotoxicity was only observed in a moderate number of patients. Therefore, the decisive question is why some patients are affected, but others not. Apart from differences in the efficacy of the individual antioxidant protection systems, a major cause may be sought in differences in the expression levels of the various CYP isoforms, which may, in some individuals, favor dealkylation, hydroxylation or epoxide formation rates and, thus, enhance naphthoquinone production.

\section{Problems of non-enzymatically oxidized metabolites}

A particular problem can arise in easily oxidizable drugs. What precisely happens in the presence of oxidants, especially free radicals, depends, of course, on the molecular properties of the compound. Small modifications of a substance may already cause substantial changes. This has been studied in some naturally occurring heterocyclic aromates, which may serve as an example. For instance, the monohydroxylated compounds serotonin and $\mathrm{N}$-acetylserotonin easily form dimers and oligomers $[1,2]$, whereas the structurally very similar, but methoxylated melatonin undergoes entirely different oxidation reactions $[2,8]$. This difference results from the fact that, upon interaction with an electron/hydrogen-abstracting radical, the hydroxylated aromate forms a C-centered organic radical that easily dimerizes, whereas the methoxylated analog forms an $\mathrm{N}$-centered radical that does not.

Dimerization or oligomerization is favored under two conditions. First, oxidative stress, which may be locally severe because of inflammation or mitochondrial malfunction, can enhance the rate of conversion. Second, administration of high doses concentrated in a pill containing an easily oxidizable drug may facilitate interactions between neighboring molecules before or shortly after uptake, an effect that disappears by dilution. The possibility of di- or oligomerization is not restricted to indoles. In particular, this may be considered in polyphenols used as food additives. Dimerization or oligomerization may be nothing more than a moderate loss of the drug, as long as the oxidation products are devoid of toxicity. However, this is not generally the case. An example may be that of another indolic compound, indole-3-propionic acid. This substance, which is a very effective scavenger of free radicals [9], had been considered as an antioxidant drug to be applied in humans. However, this compound that contains, apart from the side chain at atom 3 , no other substitution undergoes the dimerization reaction in a similar way as known from serotonin and $\mathrm{N}$-acetylserotonin. Additional oxidation reactions are not unlikely. A relatively hydrophobic, highly toxic product was detected in several experiments and already formed in the extracellular environment, especially at slightly alkaline $\mathrm{pH}$ [10]. In preclinical studies using aquatic organisms with high oxidative metabolism, the toxicity of the product formed from indole-3-propionic acid was discovered in protection experiments, in which indole3-propionic acid first behaved in a protective way, but, after a while, turned into a lethally toxic metabolite [10]. Toxicity in rodents has laterbeen observed, but the details have never been published. Suitability for treating humans has to be denied. The lesson from these findings is that easily oxidizable drugs should, for reasons of caution, not be too much concentrated in a pill. To a certain extent, this may be achieved by diluting the drug by carrier substances to avoid too many contacts between drug 
molecules. Moreover, it is important to be aware of this problem already during chemical production of the drug and later during storage. In the case of indole-3-propionic acid, the toxic compound was detected in various commercial preparations [10].

\section{Unexpected metabolite properties}

Drug metabolites may either retain or gain pharmacological properties. The demethylated agomelatine metaboliteN-[2-[7hydroxynaphth-1-yl) ethyl]acetamide, which is more serotoninlike than the parent compound, not surprisingly binds to 5 -HT2C serotonin receptors $[5,11]$. This property is shared by the parent compound, which is known to be a 5-HT2C antagonist, but the relevanceof the metabolite to agomelatine's overall actions has not been reported in detail.

An unexpected property was observed in a main metabolite of another non-indolic melatonergic agonist, ramelteon $\{=(\mathrm{S})$ $\mathrm{N}$-[2-(1,6,7,8-tetrahydro-2H-indeno[5,4-b]furan-8-yl)ethyl] propionamide (CAS 196597-26-9)\}. This metabolite, in the literature referred to as M-II, is formed by CYP1A2 and differs from the parent compound only by a hydroxyl group in the aliphatic chain, in which the propionamide residue has been changed toa 2-hydroxypropionamide [12-14]. Not surprisingly, this metabolite has retained some melatonergic activity, in the range of $10 \%$ compared to ramelteon.However, this loss of activity is compensated by a considerably longer persistence in the blood, which results in 20- to 100 -fold (median 30-fold) higher levels than those obtained with the parent compound. In the balance, the metabolite M-II contributes substantially to the overall activity of a ramelteon pill, especially in terms of duration of action [12-14]. Insofar, the outcome of this effect can be judged to be favorable. However, these findings illustrate that the persistence of metabolites in the blood is not per se unimportant, but can contribute to the pharmacology of a drug.

In terms of both activity and toxicology, the lipophilicity of drugs can be decisive. This may not only concern the distribution in the blood, but even more tissue retention. In the last years, the role of drug lipophilicity has received increasing attention, especially in terms of hepatotoxicity $[15,16]$. Usually, metabolism is seen as a means of reducing lipophilicity, e.g., by hydroxylation and conjugation. However, this is not generally the case, and the consequences of increased lipophilicity in metabolites have frequently remained unconsidered. A systematic review has summarized several cases of metabolites with higher lipophilicity than the parent compounds, changes that result, in the most plausible way, from decarboxylation [17]. However, an increase of lipophilicity can also be caused by entirely different reactions. An impressive example is that of the alkaloid berberine \{5,6-dihydro-9,10-dimethoxybenzo[g]-1,3-benzodioxolo[5,6-a] quinolizinium (CAS 2086-83-1)\}. This drug has been used for treating several diseases including type 2 diabetes and digestive problems such as SIBO (small intestine bacterial overgrowth) and related symptoms. This dimethoxylated compound carries a positive charge at its $\mathrm{N}$-atom. Metabolic dealkylation in position 9 of the ring system results in the formation of the main metabolite berberrubine \{5,6-dihydro-9-hydroxy-10methoxybenzo[g]-1,3-benzodioxolo[5,6-a]quinolizinium (CAS 15401-69-1)\} [18], a compound to which anti-cancer properties have been ascribed. Despite the expectancy of decreasing lipophilicity by demethylation, the isolated metabolite turned out to be more lipophilic. The explanation is given by conversion of the enol, as formed by demethylation, to an uncharged keto tautomer with quinoid structure [18].

\section{Molecular complexity of drugs and their consequences to metabolism}

Of course, substantial differences exist between drugs concerning the number of possible metabolites. Although no fixed rule can be deduced from the degree of molecular complexity of a drug, a tendency of potential importance can be discerned. In brief, the higher the complexity is, the higher will be, at least tendentially, the likelihood for a larger number of metabolites. This may, however, become incorrect if the primary metabolites are multiply accessible to additional metabolic reactions, including non-enzymatic reactions. An example for a multitude of molecules formed from a rather simple compound is melatonin [19-21]. The main metabolite is 6-hydroxymelatonin, which is further conjugated to 6-sulfatoxymelatonin, but a large number of additional metabolites with sometimes biological activitiesis also formed. This multitude of products can be attributed to the high reactivity of melatonin as well asof its oxidatively formed metabolites.

Nevertheless, the larger and the more complex a compound is, the higher is presumably the number of sites for metabolic modification. This seems to be especially the case when several ring systems are connected by short aliphatic brigdes. Moreover, the presence of nitrogen and oxygen atoms in the ring systems and aliphatic chains of more than two atoms (e.g., propionyl residues) can increase the number of metabolites. The melatonergic drug tasimelteon $\{(1 \mathrm{R}, 2 \mathrm{R})-\mathrm{N}-[2-(2,3-$ dihydrobenzofuran-4-yl)cyclopropylmethyl]propionamide (CAS 609799-22-6)\} may serve as an example, in which the benzofuran double ring system is connected to a cyclopropane ring, followed by an aliphatic chain. Hydroxylations are possible in the benzene, furan and cyclopropane rings and also in the aliphatic chain; moreover, glucuronidation at the newly formed hydroxyl groups, dehydrogenation at the cyclopropane and diol formation at the side chain carbonyl site are possible $[8,14]$. Moreover, oxidative ring cleavage of the furan moiety seems likely, as it is known from benzo- or indenofuran structures in other drugs, as demonstrated, e.g., in ramelteon, in which the furan is cleaved into a carboxyl group and a hydroxyethyl residue. Beginning with the difficulties of identifying all relevant metabolites of such a complex drug, the larger problem is that of characterizing the properties of all of them, especially with regard to possible toxicity. 


\section{Novel Approaches in Drug Designing \& Development}

Molecular complexity can be particular problem in multifunctional drugs, which combine entirely different residues in one molecule, in order to act at multiple receptors or to associate receptor binding with antioxidant properties. The construction of such multifunctional drugs is perceived by some chemists as a challenge with promising outcome. However, these compounds usually have reduced receptor affinity and, therefore, require elevated doses. Moreover, the number of possible metabolites is typically increased, a property that becomes more severe because of the higher doses. Therefore, thorough evaluation of metabolite formation and their possible toxicity should be regarded as a strict requirement. As summarized elsewhere [22], numerous multifunctional drugs have been developed for the treatment of Alzheimer's disease and tested in respective preclinical models. However, accompanying studies on metabolism and toxicity are frequently missing [22].

\section{Conclusion}

As illustrated by the examples mentioned above, drug metabolism and identification of eventual metabolite toxicity are necessities in drug development. Some of the possibilities can already be deduced from the molecular structure of the respective drug, as in the case of agomelatine. Strategies of predicting metabolic fates, including suitable algorithms, are highly recommended. In part, some metabolic routes can be identified in the course of Ames tests with metabolic activation by hepatic extracts. In these cases, genotoxicity may be related to oxidotoxicity. However, the absence of revertants in the Ames test does not allow the conclusion on nontoxicity. Other toxic effects different from induction of oxidative stress may still be possible and might occur via signaling mechanisms. The possibilities of interfering with receptors, ion channels and transcription factors are immense and will not be detected by tests designed for genotoxicity. With higher complexity of a drug, the likelihood for a larger number of metabolites is, on the average, increased, although most of them may occur only in low quantities. However, a low quantity should not be confused with irrelevance. The presence in minor amounts may also reflect a high reactivity that leads to rapid consumption of this metabolite, but may be involved in damage to biomolecules. Consequently, the concentration can be less important than the rate of generation, if the metabolite is readily converted. Therefore, the abundance of single main metabolites has not to be overrated. It is not sufficient to only identify the major metabolites, since minor products that are easily overlooked in a superficial screen may still cause toxicity. The lesson from quinone formation is that small amounts of a metabolite may suffice for considerable effects, especially when redox cycling is driven by a reductant such as ascorbic acid.

\section{References}

1. Huether G, Fettkötter I, Keilhoff G, Wolf G (1997) Serotonin acts as a radical scavenger and is oxidized to a dimer during the respiratory burst of activated microglia. J Neurochem 69(5): 2096-2101.

2. Behrends A, Riediger S, Grube S, Poeggeler B, Haldar C, et al. (2007)
Photocatalytic mechanisms of indoleamine destruction by the quinalphos metabolite 2-hydroxyquinoxaline: A study on melatonin and its precursors serotonin and $\mathrm{N}$-acetylserotonin. J Environ Sci Health B 42(6): 599-606.

3. Hare DJ, Double KL (2016) Iron and dopamine: a toxic couple. Brain 139(Pt 4): 1026-1035.

4. Hardeland R (2014) Agomelatine and the risk of hepatotoxicity. J Symptoms Signs 3(5): 341-346.

5. Hardeland R, Poeggeler B, Srinivasan V, Trakht I, Pandi-Perumal SR, etal. (2008) Melatonergic drugs in clinical practice. Arzneimittelforschung 58(1): $1-10$.

6. Gahr M, Freudenmann RW, Connemann BJ, Hiemke C, SchönfeldtLecuona C (2013) Agomelatine and hepatotoxicity: implications of cumulated data derived from spontaneous reports of adverse drug reactions. Pharmacopsychiatry 46(6): 214-220.

7. Štuhec M (2013) Agomelatine-induced hepatotoxicity. Wiener KlinWschr 125: 225-226.

8. Hardeland R (2009) New approaches in the management of insomnia: weighing the advantages of prolonged release melatonin and synthetic melatoninergic agonists. Neuropsychiatr Dis Treat 5: 341-354.

9. Poeggeler B, Pappolla MA, Hardeland R, Rassoulpour A, Hodgkins PS, et al. (1999) Indole-3-propionate: A potent hydroxyl radical scavenger in rat brain. Brain Res 815(2): 382-388.

10. Holst S, Poeggeler B, Hardeland R (2001) A note on the conditions of toxicity and non-toxicity of indole-3-propionic acid. In: Hardeland R, (Edt.), Actions and Redox Properties of Melatonin and Other Aromatic Amino Acid Metabolites, Cuvillier, Göttingen, pp. 91-95.

11. Chagraoui A, Protais P, Filloux T, Mocaër E (2003) Agomelatine (S 20098) antagonizes the penile erections induced by the stimulation of 5-HT2C receptors in Wistar rats. Psychopharmacology (Berl) 170(1): $17-22$

12. Karim A, Tolbert D, Cao C (2006) Disposition kinetics and tolerance of escalating single doses of ramelteon, a high affinity MT1 and MT2 melatonin receptor agonist indicated for the treatment of insomnia. J ClinPharmacol 46(2): 140-148.

13. Pandi-Perumal SR, Srinivasan V, Poeggeler B, Hardeland R, Cardinali DP (2007) Drug insight: the use of melatonergic agonists for the treatment of insomnia - focus on ramelteon. Nat ClinPractNeurol 3(4): 221-228.

14. Hardeland R (2016) Melatonin and synthetic melatoninergic agonists in psychiatric and age-associated disorders: successful and unsuccessful approaches. Curr Pharm Des 22(8): 1086-1101.

15. Chen M, Borlak J, Tong WA (2016) Model to predict severity of druginduced liver injury in humans. Hepatology 64(3): 931-940.

16. McEuen K, Borlak J, Tong W, Chen M (2017) Associations of drug lipophilicity and extent of metabolism with drug-induced liver injury. Int J Mol Sci 18(7): E1335.

17. Hartyánszky I, Kalász H, Adeghate E, Gulyás Z, Hasan MY, et al. (2012) Active metabolites resulting from decarboxylation, reduction and ester hydrolysis of parent drugs. Curr Drug Metab 13(6): 835-862.

18. Spinozzi S, Colliva C, Camborata C, Roberti M, Ianni C, et al. (2014) Berberine and its metabolites: relationship between physicochemical properties and plasma levels after administration to human subjects. J Nat Prod 77(4): 766-772.

19. Tan D-X, Manchester LC, Terron MP, Flores LJ, Reiter RJ (2007) One molecule, many derivatives: a never-ending interaction of melatonin with reactive oxygen and nitrogen species? J Pineal Res 42(1): 28-42.

20. Hardeland R (2008) Melatonin, hormone of darkness and more occurrence, control mechanisms, actions and bioactive metabolites. Cell Mol Life Sci 65(13): 2001-2018. 
21. Hardeland R, Tan D-X, Reiter RJ (2009) Kynuramines, metabolites of melatonin and other indoles: the resurrection of an almost forgotten class of biogenic amines. J Pineal Res 47(2): 109-126.

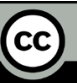

22. Hardeland R (2011) Cognitive enhancers in moderate to severe Alzheimer's disease. Clin Med Insights Ther 3: 459-476.

Your next submission with Juniper Publishers will reach you the below assets

- Quality Editorial service

- Swift Peer Review

- Reprints availability

- E-prints Service

- Manuscript Podcast for convenient understanding

- Global attainment for your research

- Manuscript accessibility in different formats

( Pdf, E-pub, Full Text, Audio)

- Unceasing customer service

Track the below URL for one-step submission https://juniperpublishers.com/online-submission.php 